\title{
Executive Functioning in Gambling Disorder: Cognitive Profiles and Associations with Clinical Outcomes
}

\author{
Antonio Verdejo-Garcia ${ }^{1,2}$ • Victoria Manning ${ }^{2,3}$
}

Published online: 9 July 2015

(C) Springer International Publishing AG 2015

\begin{abstract}
Gambling disorder shares neurobiological and clinical symptoms with substance use disorders; however, it remains unclear if they share executive functioning deficits that compromise gambling treatments. In this review article, findings from the resurgence in clinical and cognitive neuroscience studies in the past 3 years are considered and their role in clinical decision-making re-appraised. In contrast to early findings, novel studies are suggestive of preserved general executive functions (i.e. working memory, planning, rule shifting), but lend further support for deficits of response inhibition, reward-related switching and value-based decisionmaking, although evidence of their impact on treatment outcome remains limited. The hallmark characteristic of disordered gambling "cognitive impulsivity" may reflect an underlying core alteration in value coding. Increased attention to wins and blunted attention to losses may serve to maintain maladaptive gambling behaviours (e.g. loss chasing), thus the effectiveness of interventions targeting faulty brain value systems (e.g. cognitive bias modification) warrants investigation with this population.
\end{abstract}

Keywords Cognitive impulsivity · Decision-making · Gambling $\cdot$ Value coding $\cdot$ Sensitivity to gains $\cdot$ Treatment outcomes

This article is part of the Topical Collection on Gambling

Antonio Verdejo-Garcia

Antonio.Verdejo@monash.edu

1 School of Psychological Sciences, Monash University, 3800 Wellington Rd., Melbourne, Australia

2 Turning Point Alcohol \& Drug Centre, Melbourne, Australia

3 Eastern Health Clinical School, Monash University, Melbourne, Australia

\section{Introduction}

Gambling disorder is now classified as an addictive disorder, based on the overlap with substance use disorders in terms of neurobiology and clinical manifestations such as preoccupation, loss of control and continued engagement in the behaviour despite negative consequences and relapses [1]. In the last two decades, clinical and cognitive neuroscience studies have revealed that individuals with substance use disorders exhibit significant cognitive deficits encompassing executive functions, decision-making and reward processing [2]. Moreover, deficits in executive functions and decision-making have been linked to poorer treatment outcomes and higher rates of drug relapse [3-5]. These cognitive findings are fostering a paradigm shift in substance addiction treatment whereby cognitive profiles could be effectively employed to predict treatment response [6], and targeted by cognitive enhancement interventions to reduce the liability to drug relapse [7]. Since gambling disorder shares neurobiology and clinical symptoms with substance use disorders, two relevant questions remain unresolved: is gambling disorder (i.e. a behavioural addiction) associated with similar cognitive deficits to those found in substance addictions, and if so, are these cognitive deficits relevant to predict gambling treatment outcomes?

Early cognitive studies in pathological gambling - now gambling disorder-showed that compared to healthy controls, they exhibit deficits in executive functions (i.e. planning, flexibility) and cognitive impulsivity (i.e. response inhibition, reflection impulsivity and decision-making) [8-10]. In fact, the performance of pathological gamblers in cognitive tests tapping on these domains was similar to that found in alcohol-dependent individuals. These early studies highlighted the relevance of cognitive profiles for the development and recovery of gambling disorder. However, more research was needed to build a case for the consideration of cognitive 
findings in gambling-related policy and clinical practice. This review will capitalise on novel evidence (i.e. cognitive studies published in the last 3 years) to reassess our appraisal of the key questions described above; in summary: is gambling disorder linked to cognitive deficits, and does it have a role to play in clinical decision-making? In the first section, we review the studies examining the cognitive profile linked to gambling disorder, and in the second section, the association between gambling disorder-related cognitive profiles and treatment outcomes. We conclude with some tentative recommendations to translate cognitive findings into effective interventions for individuals with gambling disorder.

\section{The Cognitive Profile Linked to Gambling Disorder}

Current clinical and cognitive neuroscience research has incorporated novel approaches to refine cognitive profiling in individuals with gambling disorder (GD). These approaches include the administration of cognitive tasks and batteries capable of dissociating processes traditionally grouped under the same domain (i.e. different aspects of executive functions), the application of cognitive modelling to identify key processes within complex cognitive tasks (e.g. the Iowa Gambling Task) and the inclusion of appropriately matched control groups that are relevant to disentangle the cognitive profile of GD from other factors (e.g. IQ, years of education) or common comorbidities such as substance use disorders. In this section we primarily review studies utilising these novel approaches to cognitive assessment and profiling. Moreover, we occasionally draw upon neuroimaging evidence to enrich neurosciencebased interpretations of cognitive findings, since studies have reported differences in neural activation patterns between GD and healthy controls in the absence of behavioural differences in executive functioning test performance $[11,12]$.

The "classic cognitive profile" attributed to GD based on early studies encompasses deficits in executive functions, decision-making (including impulsive decision styles such as increased reflection impulsivity and higher delay discounting) and deficits in reinforcement learning [8-10]. Executive functions refer to a collection of top-level cognitive skills implicated in goal-oriented behaviour, including working memory (i.e. holding information on line), response inhibition (i.e. stopping prepotent responses) and flexibility (i.e. switching unproductive response patterns) [13] and as such studies frequently adopt a battery of objective neuropsychological tests that tap in to multiple executive functioning domains. In doing so, novel studies provide clarification of the executive skills altered in GD. For instance, recent studies broadly indicate normal performance in tests of verbal and spatial working memory [14••, 15-18] assessed using the NBack, Letter-Number Sequencing and Spatial Span working memory tasks [17] with unimpaired planning and organisational skills also reported (Stockings of Cambridge) [17]. With regard to response inhibition, novel studies broadly accord with earlier reports [9] showing increased stop-signal inhibition errors in GD individuals compared to healthy controls and also compared to problem gamblers not meeting criteria for GD [19••]; however, others have failed to find differences on a GoStop impulsivity paradigm [16]. In terms of flexibility, individuals with GD do not exhibit perseverative errors on the Wisconsin Card Sorting Test compared to healthy controls [20, 21]. In fact, Boog et al. [21] contrasted performance of GD and healthy controls in two tests tapping on different aspects of flexibility: switching an arbitrary rule (Wisconsin Card Sorting Test) versus switching previously rewarded choices (Probabilistic Reversal Learning Task). Controls were found to only outperform GD individuals in the reward-based Probabilistic Reversal Learning Task, thus suggesting a motivational contribution to poor flexibility in GD, rather than a general cognitive inflexibility problem. The cognitive literature on executive functions in GD aligns with neuroimaging findings indicating that GD is associated with functional abnormalities in the dorsomedial prefrontal cortex, implicated in reinforcement learning, but with preserved function of the dorsolateral prefrontal cortex, implicated in working memory [12].

In the decision-making literature (including impulsive decision styles), recent studies have also contributed to the identification of the key processes contributing to poor choices in GD. Several studies report deficits in decision-making as demonstrated by performance on the IGT [16, 22, 23]. Cognitive decision-making theory posits a dissociation between decisions under ambiguity (when outcomes are not explicit) and decisions under risk (when outcomes are explicit) [24], and individuals with GD have demonstrated deficits in tests of both modalities [15, 22]. However, the application of cognitive modelling approaches has revealed that the decisionmaking deficits of GD can be effectively accounted for by a core alteration in the motivational processing of gains and losses [25, 26••]. Specifically, individuals with GD display higher attention to gains and lower attention to losses during decision-making tests, thus fuelling choices that are (or have been) linked to reward, regardless of changes in expected value. Moreover, increased attention to gains and blunted attention to losses are even more pronounced in more severe forms of GD [25, 26••]. The "attention to gains" account may also explain GD deficits in delay discounting tests, in which they show a higher preference for immediate rewards, which are arguably perceived as more salient relative to delayed offers [27]. Interestingly, it has been speculated that this "attention to gains" account may also contribute to explain GD deficits in other cognitive impulsivity tests that create a conflict between approach and avoidance responses (i.e. stop signal or reflection impulsivity). Since approach tendencies are inherently intertwined with reward, and avoidance 
tendencies inherently intertwined with punishment, individuals with GD may find it more challenging to stop "goreward" responses due to deficient coding of reward and punishment value [28•]. Given their demonstrated impact reducing these approach/avoidance biases among alcoholdependent drinkers [29], there may be a role for cognitive bias modification training programs in GD, as we describe in the "Cognitive Profiles, Course of Pathology and Clinical Outcomes" section below. Altogether, the cognitive literature on decision-making in GD maps on to neuroimaging findings indicating that GD is associated with functional alterations in the brain reward coding system (i.e. the striatum and functionally connected regions such as the orbitofrontal cortex and the subgenual anterior cingulate cortex). These deficits are characterised by sensitised response of the striatum to cues signalling monetary gains versus natural reinforcers [30], and by non-categorical coding of reward feedback, i.e. rewards of different magnitude and even near misses are uniformly coded [31,32].

The findings from the above studies described generally hold true for disordered gamblers in the context of clinical comorbidities. With regard to executive functions, deficits in response inhibition and response switching after reversal of reward schedule are comparable in GD and substance use disorders (SUD) [14・•,33], although there is evidence of negative correlations between switching performance and general estimates of psychological problems or symptoms of psychopathology symptoms on the Derogatis' symptom checklistin GD [21]. With regard to decision-making, studies have consistently showed that deficits in value-based decisionmaking are similar in individuals with GD alone and individuals with GD and SUD comorbidities [18, 34, 35]. Nonetheless, concurrent alcohol and tobacco use are associated with higher betting in GD [35], a finding that, if consistently replicated, may have policy implications in terms of availability of alcohol and tobacco products in gambling settings. Intriguingly, delay discounting deficits are more pronounced in GD compared to both SUDs and SUDs with complex clinical comorbidities [36], thus supporting the notion that altered value-based choice is the key cognitive hallmark of GD.

\section{Cognitive Profiles, Course of Pathology and Clinical Outcomes}

Intuitively, the executive functioning deficits common among individuals with GD (i.e. poor response inhibition, high delay discounting and aberrant reward-based learning) are likely to maintain well-established gambling behaviour patterns and could conceivably diminish treatment efficacy as literature suggests they do with substance use disorders [37-39]. However, there remains a paucity of research on the impact of cognitive impairment on the both the development of disordered gambling and treatment response. In a small sample of outpatient lifetime pathological gamblers, neither Iowa Gambling Task, Delay Discounting Task nor Stroop Gambling Task performance differentiated those who had abstained from gambling $(n=9)$ and those who had relapsed at 1-year follow-up $(n=13)$ [40•], suggesting that impulsivity and decision-making does not influence long-term outcome. These findings are supportive of earlier studies [41, 42], though the later study did find an association between the absence of gambling problems at 1-year and performance on the stop-signal task and performance in a card-playing task, suggesting that disinhibition and perseveration for reward relate to treatment outcome. Recently, Giorgetta et al. [43] examined differences in loss aversion, measured using a flipcoin task and reported that pathological gamblers after longterm treatment (more than 18 months) demonstrated significantly greater loss aversion to both pathological gamblers who had received less than 6 months of treatment and healthy controls. However, this difference was limited to gambles where the potential gain was outweighed by the potential loss, suggesting that as patients recover, they demonstrate greater sensitivity to losses. A strength of this study was the recruitment of demographically matched controls that themselves gamble, and the findings suggest that loss aversion increases in response to clinical treatment. However, since gambling status of the early and long-term treatment groups GD patient groups was not reported and gambling severity score (SOGS) failed to differ, it is difficult to interpret the implications of this study in terms of the reported treatment response and clinical outcome. Unfortunately, there has been equally scant attention to the impact of cognitive functioning on treatment outcomes other than gambling status (e.g. psychosocial functioning and treatment completion). Giorgetta et al. [43] found that despite greater loss aversion and acceptance of fewer gambles (with a positive expected value) among late stage relative to early stage treatment, anxiety and depression scores did not differ. Similarly, from an extensive battery of executive functioning tests, AlvarezMoya et al. [41] found that a lower number of advantageous choices on the IGT-EFGH (a measure of sensitivity to gains and losses) emerged as the sole predictor of treatment dropout.

Discrepant findings in the cognition and clinical outcomes field could be explained by a number of methodological factors, many of which have been previously described in relation to neuropsychological research on persons with substance use disorders [44]. This includes differences in the cognitive domain assessed, the nature and complexity of the neuropsychological task used to assess that domain and performance parameter adopted. Studies also differ in how treatment outcome is operationalised (e.g. abstinence from all gambling forms or only the problem gambling activity or the absence of gambling problems despite slips or lapses versus continuous abstinence) as well the timing of assessment, which relates to the stage of recovery, treatment duration or post-treatment 
follow-up period. Sampling differences, e.g. treatment seeking versus community sampled problem gamblers and their heterogeneity, e.g. strategic versus non-strategic gamblers, and co-occurring substance or other impulse control disorders that can also impact on cognitive functioning [26••], further hamper comparisons across studies. Finally, studies typically recruit small samples of around 20 individuals with GD and an equivalent number of controls who have fulfilled strict inclusion/exclusion criteria. This means studies are often insufficiently powered to detect small effects sizes and samples are not representative of the broader GD population.

\section{Conclusion}

This targeted review of the novel cognitive literature in GD shows that (1) GD is linked to cognitive deficits in response inhibition, reward-related switching and value-based decision-making, but with preserved working memory, planning and rule shifting, and (2) response inhibition and rewardbased decision-making are the only EF deficits associated with the clinical outcomes of GD treatment so far; however, research in this area remains limited.

Collectively, the new wave of cognitive studies indicates that deficits in value-laden response inhibition, reversal learning and decision-making are very much the cognitive imprint of GD. On the basis of these findings and in combination with neuroimaging findings [30-32], we speculatively propose that this set of deficits may be underlain by a core alteration in the coding of the motivational value of stimuli, whereby high attention to gains and low attention to losses primes "go" versus "stop" responses in response inhibition tests, and choices linked to superficially salient rewards in reversal learning, decision-making and delay discounting tests. Therefore, this set of deficits could be best characterised by core alterations in the coding of motivational value, which have a fluid impact on high-level decision-making systems [45]. More research is required to achieve a better understanding of the role of gambling severity and gambling-related clinical comorbidities in the cognitive profile of GD. Recent studies also consistently indicate that the aforementioned executive functioning deficits are more pronounced in more severe forms of GD. Early studies proposed that gambling modality (slot machine versus strategic gambling) was a critical factor contributing to cognitive deficits, but this assumption is somehow obscured by the observation that slot machine gamblers often demonstrate greater problem gambling symptom severity and have more clinical comorbidities than generally higher functioning strategic gamblers [26••]. A more dimensional approach to gambling severity, whereby multiple sources of risk are cumulatively factored in (i.e. genetic, environmental and disorder-specific) will likely contribute to a better understanding of cognitive phenotypes in GD.
In relation to the association between cognitive profiles and clinical outcomes, the novel findings are predominantly negative, but new research is scarce and arguably underpowered; thus, positive findings from early studies may still be valid. In fact, based on our interpretation of the cognitive literature and evidences from qualitative studies [46], we speculate that future research utilising motivationally laden choice tests should be able to identify cognitive markers of treatment failure. The application of cognitive modelling to measure the key motivational processes that dynamically evolve during value-based decision-making, and the application of machine learning techniques for pattern classification may substantially improve the predictive validity of these cognitive studies. The critical question that remains unanswered is whether these cognitive findings may inform development of novel therapeutic interventions to reduce relapse in GD. Since the key cognitive findings (i.e. deficits in response inhibition, reward-related switching and value-based decision-making) have been consistently replicated, and there is strong neuroscience evidence that links these cognitive deficits to alterations in value coding $[30,31]$, it is reasonable to propose that neurocognitive interventions targeting implicit motivational processes, e.g. reducing attention and approach bias via cognitive bias modification (CBM) [29] may reduce the liability to gambling relapse. Moreover, CBM has shown to effectively reduce the coding of salient cues in the brain reward system [47], and therefore, it may contribute to restore the balance in the value system. Using a computerised training program, disordered gamblers could be trained to implicitly avoid gambling-related images (e.g. EGMs) and approach non-gambling-related images tailored to individual differences. $\mathrm{CBM}$ and other cognitive interventions targeting brain value systems are examples of innovative approaches driven by neurobiological, neuropsychological and neuroscience findings and so their application to individuals with gambling disorders should be a priority for future treatment outcome research.

\section{Compliance with Ethics Guidelines}

Conflict of Interest Antonio Verdejo-Garcia and Victoria Manning declare that they have no conflict of interest.

Human and Animal Rights and Informed Consent This article contains information about studies with human subjects performed by the authors; these studies were conducted with the approval of the relevant Human Research Ethics committees and included written informed consent from participants. This article does not contain any studies with animal subjects performed by any of the authors. 


\section{References}

Papers of particular interest, published recently, have been highlighted as:

- Of importance

- Of major importance

1. American Psychiatric Association. Diagnostic and statistical manual of mental disorders. 5th ed. Arlington: American Psychiatric Publishing; 2013.

2. Fernandez-Serrano MJ, Perez-Garcia M, Verdejo-Garcia A. What are the specific vs. generalized effects of drugs of abuse on neuropsychological performance? Neurosci Biobehav Rev. 2011;35(3): 377-406.

3. Stevens L, Goudriaan AE, Verdejo-Garcia A, Dom G, Roeyers H, Vanderplasschen W. Impulsive choice predicts short-term relapse in substance-dependent individuals attending an in-patient detoxification programme. Psychol Med. 2015;2:1-11.

4. Streeter CC, Terhune DB, Whitfield TH, Gruber S, Sarid-Segal O, Silveri MM, et al. Performance on the Stroop predicts treatment compliance in cocaine-dependent individuals. Neuropsychopharmacol Off Publ Am Coll Neuropsychopharmacol. 2008;33(4):827-36.

5. Verdejo-Garcia A, Albein-Urios N, Martinez-Gonzalez JM, Civit E, de la Torre R, Lozano O. Decision-making impairment predicts 3month hair-indexed cocaine relapse. Psychopharmacology. 2014;231(21):4179-87.

6. Stevens L, Verdejo-Garcia A, Goudriaan AE, Roeyers H, Dom G, Vanderplasschen W. Impulsivity as a vulnerability factor for poor addiction treatment outcomes: a review of neurocognitive findings among individuals with substance use disorders. J Subst Abus Treat. 2014;47(1):58-72.

7. Bates ME, Buckman JF, Nguyen TT. A role for cognitive rehabilitation in increasing the effectiveness of treatment for alcohol use disorders. Neuropsychol Rev. 2013;23(1):27-47.

8. Goudriaan AE, Oosterlaan J, de Beurs E, van den Brink W. Decision making in pathological gambling: a comparison between pathological gamblers, alcohol dependents, persons with Tourette syndrome, and normal controls. Brain Res Cogn Brain Res. 2005;23(1):137-51.

9. Goudriaan AE, Oosterlaan J, de Beurs E, van den Brink W. Neurocognitive functions in pathological gambling: a comparison with alcohol dependence, Tourette syndrome and normal controls. Addiction. 2006;101(4):534-47.

10. Lawrence AJ, Luty J, Bogdan NA, Sahakian BJ, Clark L. Impulsivity and response inhibition in alcohol dependence and problem gambling. Psychopharmacology. 2009;207(1):16372.

11. de Ruiter MB, Oosterlaan J, Veltman DJ, van den Brink W, Goudriaan AE. Similar hyporesponsiveness of the dorsomedial prefrontal cortex in problem gamblers and heavy smokers during an inhibitory control task. Drug Alcohol Depend. 2012;121(1-2):819.

12. Verdejo-Garcia A, Clark L, Verdejo-Roman J, Albein-Urios N, Martinez-Gonzalez JM, Gutierrez B, Soriano-Mas C. Neural substrates of cognitive flexibility in stimulant and gambling addictions. Br J Psychiatry. in press.

13. Verdejo-Garcia A, Perez-Garcia M. Profile of executive deficits in cocaine and heroin polysubstance users: common and differential effects on separate executive components. Psychopharmacology. 2007;190(4):517-30.

14.• Albein-Urios N, Martinez-Gonzalez JM, Lozano O, Clark L, Verdejo-Garcia A. Comparison of impulsivity and working memory in cocaine addiction and pathological gambling: Implications for cocaine-induced neurotoxicity. Drug Alcohol Depend.
2012;126(1-2):1-6. Demonstrates that pathological gamblers (PGs) and cocaine dependents have common deficits in response inhibition. Conversely, PGs have normal working memory performance in an $n$-back task. Illustrates specificity of executive function deficits in gambling disorder.

15. Brevers D, Cleeremans A, Goudriaan AE, Bechara A, Kornreich C, Verbanck $\mathrm{P}$, et al. Decision making under ambiguity but not under risk is related to problem gambling severity. Psychiatry Res. 2012;200(2-3):568-74.

16. Ledgerwood DM, Orr ES, Kaploun KA, Milosevic A, Frisch GR, Rupcich N, et al. Executive function in pathological gamblers and healthy controls. J Gambl Stud. 2012;28(1):89-103.

17. Manning V, Teoh HC, Guo S, Wong KE, Li TK. Executive functioning in Asian pathological gamblers. Int Gambl Stud. 2013;13(3):403-16.

18. Yan WS, Li YH, Xiao L, Zhu N, Bechara A, Sui N. Working memory and affective decision-making in addiction: a neurocognitive comparison between heroin addicts, pathological gamblers and healthy controls. Drug Alcohol Depend. 2014;134: 194-200.

19.• Brevers D, Cleeremans A, Verbruggen F, Bechara A, Kornreich C, Verbanck P, et al. Impulsive action but not impulsive choice determines problem gambling severity. PloS one. 2012;7(11):e50647. Demonstrates that deficits in stop signal response inhibition differentiate pathological gamblers from both problem gamblers and healthy controls.

20. Hur JW, Shin N, Kim SN, Jang JH, Choi JS, Shin YC, et al. Do pathological gambling and obsessive-compulsive disorder overlap? a neurocognitive perspective. CNS Spectr. 2012;17(4):207-13.

21. Boog M, Hoppener P, Wetering BJVD, Goudriaan AE, Boog MC, Franken IH. Cognitive inflexibility in gamblers is primarily present in reward-related decision making. Front Hum Neurosci. 2014;8: 569.

22. Kraplin A, Dshemuchadse M, Behrendt S, Scherbaum S, Goschke $\mathrm{T}$, Buhringer G. Dysfunctional decision-making in pathological gambling: pattern specificity and the role of impulsivity. Psychiatry Res. 2014;215(3):675-82.

23. Ochoa C, Alvarez-Moya EM, Penelo E, Aymami MN, GomezPena M, Fernandez-Aranda F, et al. Decision-making deficits in pathological gambling: the role of executive functions, explicit knowledge and impulsivity in relation to decisions made under ambiguity and risk. Am J Addict. 2013;22(5):492-9.

24. Bechara A. Risky business: emotion, decision-making, and addiction. J Gambling Stud Co-sponsored Natl Counc Probl Gambl Inst Stud Gambling Commer Gaming. 2003;19(1):23-51.

25. Brevers D, Koritzky G, Bechara A, Noel X. Cognitive processes underlying impaired decision-making under uncertainty in gambling disorder. Addict Behav. 2014;39(10):1533-6.

26.• Lorains FK, Dowling NA, Enticott PG, Bradshaw JL, Trueblood JS, Stout JC. Strategic and non-strategic problem gamblers differ on decision-making under risk and ambiguity. Addiction. 2014;109(7): 1128-37. Demonstrates that the decision-making deficits of pathological gamblers are underlain by hypersensitivity to gains and hyposensitivity to loss processes.

27. O'Connor DA, Meade B, Carter O, Rossiter S, Hester R. Behavioral sensitivity to reward is reduced for far objects. Psychol Sci. 2014;25(1):271-7.

28. Stevens T, Brevers D, Chambers CD, Lavric A, McLaren IP, Mertens M, et al. How does response inhibition influence decision making when gambling? J Exp Psychol Appl. 2015;21(1):15-36. Introduces the notion that response inhibition deficits in pathological gamblers (PGs) may be underlain by habituated motivational tendencies: Go responses are linked to reward and Stop responses to punishment, thus PGs find more challenging to control responses cached as rewarding. 
29. Wiers RW, Eberl C, Rinck M, Becker ES, Lindenmeyer J. Retraining automatic action tendencies changes alcoholic patients' approach bias for alcohol and improves treatment outcome. Psychol Sci. 2011;22(4):490-7.

30. Sescousse G, Barbalat G, Domenech P, Dreher JC. Imbalance in the sensitivity to different types of rewards in pathological gambling. Brain J Neurol. 2013;136(Pt 8):2527-38.

31. Clark L, Lawrence AJ, Astley-Jones F, Gray N. Gambling nearmisses enhance motivation to gamble and recruit win-related brain circuitry. Neuron. 2009;61(3):481-90.

32. van Holst RJ, Chase HW, Clark L. Striatal connectivity changes following gambling wins and near-misses: associations with gambling severity. NeuroImage Clin. 2014;5:232-9.

33. Torres A, Catena A, Candido A, Maldonado A, Megias A, Perales JC. Cocaine dependent individuals and gamblers present different associative learning anomalies in feedback-driven decision making: a behavioral and ERP study. Front Psychol. 2013;4:122.

34. Kraplin A, Buhringer G, Oosterlaan J, van den Brink W, Goschke T, Goudriaan AE. Dimensions and disorder specificity of impulsivity in pathological gambling. Addict Behav. 2014;39(11):1646-51.

35. Zois E, Kortlang N, Vollstadt-Klein S, Lemenager T, Beutel M, Mann K, et al. Decision-making deficits in patients diagnosed with disordered gambling using the Cambridge Gambling task: the effects of substance use disorder comorbidity. Brain Behav. 2014;4(4):484-94.

36. Albein-Urios N, Martinez-Gonzalez JM, Lozano O, Verdejo-Garcia A. Monetary delay discounting in gambling and cocaine dependence with personality comorbidities. Addict Behav. 2014;39(11): 1658-62.

37. Bowden-Jones H, McPhillips M, Rogers R, Hutton S, Joyce E. Risk-taking on tests sensitive to ventromedial prefrontal cortex dysfunction predicts early relapse in alcohol dependency: a pilot study. J Neuropsychiatry Clin Neurosci. 2005;17(3):417-20.

38. Passetti F, Clark L, Mehta MA, Joyce E, King M. Neuropsychological predictors of clinical outcome in opiate addiction. Drug Alcohol Depend. 2008;94(1-3):82-91.

39. Verdejo-Garcia A, Betanzos-Espinosa P, Lozano OM, VergaraMoragues E, Gonzalez-Saiz F, Fernandez-Calderon F, et al. Selfregulation and treatment retention in cocaine dependent individuals: a longitudinal study. Drug Alcohol Depend. 2012;122(1-2):142-8.

40. De Wilde B, Goudriaan A, Sabbe B, Hulstijn W, Dom G. Relapse in pathological gamblers: a pilot study on the predictive value of different impulsivity measures. J Behav Addict. 2013;2(1):23-30.

Demonstrates that even the most common deficits in GD i.e. impulsivity (Delay Discounting Task), decision-making (IGT) and attentional bias (Stroop Colour Word Test) measured on treatment entry failed to predict abstinent from relapsed gamblers one-year later.

41. Alvarez-Moya EM, Ochoa C, Jiménez-Murcia S, Aymamí MN, Gómez-Peña M, Fernández-Aranda $\mathrm{F}$, et al. Effect of executive functioning, decision-making and self-reported impulsivity on the treatment outcome of pathologic gambling. J Psychiatry Neurosci. 2011;36(3):165-75.

42. Goudriaan AE, Oosterlaan J, De Beurs E, Van Den Brink W. The role of self-reported impulsivity and reward sensitivity versus neurocognitive measures of disinhibition and decision-making in the prediction of relapse in pathological gamblers. Psychol Med. 2008;38(1):41-50.

43. Giorgetta C, Grecucci A, Rattin A, Guerreschi C, Sanfey AG, Bonini N. To play or not to play: a personal dilemma in pathological gambling. Psychiatry Res. 2014;219(3):562-9.

44. Verdejo-Garcia A, Lopez-Torrecillas F, Gimenez CO, Perez-Garcia M. Clinical implications and methodological challenges in the study of the neuropsychological correlates of cannabis, stimulant, and opioid abuse. Neuropsychol Rev. 2004;14(1):1-41.

45. Daw ND, Gershman SJ, Seymour B, Dayan P, Dolan RJ. Modelbased influences on humans' choices and striatal prediction errors. Neuron. 2011;69(6):1204-15.

46. Oakes J, Pols R, Battersby M, Lawn S, Pulvirenti M, Smith D. A focus group study of predictors of relapse in electronic gaming machine problem gambling, part 1: factors that 'push' towards relapse. J Gambl Stud. 2012;28(3):451-64.

47. Wiers CE, Stelzel C, Gladwin TE, Park SQ, Pawelczack S, Gawron $\mathrm{CK}$, et al. Effects of cognitive bias modification training on neural alcohol cue reactivity in alcohol dependence. Am J Psychiatry. 2015;172(4):335-43. 